\title{
BMJ
}

\section{Association between waiting times and short term mortality and hospital admission after departure from emergency department: population based cohort study from Ontario, Canada}

\section{Astrid Guttmann, senior scientist, ${ }^{1,2,3,4}$ Michael I Schull, senior scientist and 2010-11 Commonwealth Fund Harkness fellow, 1,4,5,6,7 Marian J Vermeulen, epidemiologist, ${ }^{1,6}$ Therese A Stukel, senior scientistt,4,6}

\begin{abstract}
Institute for Clinical Evaluative Sciences, Toronto, ON, Canada ${ }^{2}$ Divisions of Paediatric and Emergency Medicine, Hospital for Sick Children, Toronto

${ }^{3}$ Department of Paediatrics, University of Toronto, Toronto

${ }^{4}$ Department of Health Policy, Management and Evaluation, University of Toronto, Toronto

${ }^{5}$ Department of Emergency Services, Sunnybrook Health Sciences Centre, Toronto

${ }^{6}$ Clinical Epidemiology Unit, Sunnybrook Health Sciences Centre, Toronto

${ }^{7}$ Department of Medicine, University of Toronto, Toronto Correspondence to: A Guttmann, Institute for Clinical Evaluative Sciences, G-106, 2075 Bayview Ave, Toronto, ON, CanadaM4N3M5 astrid.guttmann@ices.on.ca
\end{abstract}

Cite this as: BMJ 2011;342:d2983 doi:10.1136/bmj.d2983

\section{ABSTRACT}

Objective To determine whether patients who are not admitted to hospital after attending an emergency department during shifts with long waiting times are at risk for adverse events.

Design Population based retrospective cohort study using health administrative databases.

Setting High volume emergency departments in Ontario, Canada, fiscal years 2003-7.

Participants All emergency department patients who were not admitted (seen and discharged; left without being seen).

Outcome measures Risk of adverse events (admission to hospital or death within seven days) adjusted for important characteristics of patients, shift, and hospital. Results 13934542 patients were seen and discharged and 617011 left without being seen. The risk of adverse events increased with the mean length of stay of similar patients in the same shift in the emergency department. For mean length of stay $\geq 6 v<1$ hour the adjusted odds ratio (95\% confidence interval) was 1.79 (1.24 to 2.59 ) for death and 1.95 (1.79 to 2.13 ) for admission in high acuity patients and 1.71 (1.25 to 2.35) for death and 1.66 (1.56 to 1.76 ) for admission in low acuity patients). Leaving without being seen was not associated with an increase in adverse events at the level of the patient or by annual rates of the hospital.

Conclusions Presenting to an emergency department during shifts with longer waiting times, reflected in longer mean length of stay, is associated with a greater risk in the short term of death and admission to hospital in patients who are well enough to leave the department. Patients who leave without being seen are not at higher risk of short term adverse events.

\section{INTRODUCTION}

Waiting times in emergency departments are a considerable problem in many countries. ${ }^{1-3}$ Lengthening waiting times and associated crowding have led the Institute of Medicine to describe American emergency departments as "nearing the breaking point." ${ }^{4}$ To deal with the problem of waiting times, policymakers in Canada, Australia, and England ${ }^{5-7}$ have instituted health reforms that include setting targets for the time patients spend in the department. Recently, however, the British government has rescinded National Health Service (NHS) emergency department targets for lack of "clinical justification."

The evidence on the impact of waiting times has focused on high acuity patients. Long waiting times are associated with delays in time sensitive treatments for serious conditions typically requiring admission to hospital. ${ }^{910}$ About $85 \%$ of patients attending an emergency department, however, go home after their visit, ${ }^{11}$ and whether waiting times adversely affect their outcomes is unknown. Long waiting times can delay every stage of the visit, from initial assessment to treatment to final decision making to admission or discharge (patient disposition) and can alter clinicians' routines and decision making. ${ }^{12}$ For patients, the frustration with long waits can cause up to $10 \%$ to leave without being seen (that is, before seeing a physician, without a diagnosis or treatment). ${ }^{12}$ Hence, long waiting times can alter the behaviour of both staff and patients and lead to potentially adverse consequences.

\section{Objectives}

The National Quality Forum in the United States has endorsed the use of annual rates of patients who leave hospital without being seen and lengths of stay in emergency departments as measures of quality and efficiency. ${ }^{13}$ We examined whether these factors are associated with an increased risk of death and admission to hospital within seven days in patients who are not admitted. We focused on patients who were not admitted to hospital because they form the large majority of patients and are thought to be less seriously ill and therefore rarely studied and subsequent outcomes are more directly associated with care in the emergency department compared with admitted patients. We determined the relative risk of adverse events when patients present to departments with longer waiting 
times or with higher rates of patients who leave without being seen; in addition we compared outcomes for patients who leave without being seen with those who are seen by a physician and discharged. We hypothesised that patients in departments with longer shift waiting times are at increased risk of adverse outcomes, with higher acuity patients being at particular risk; patients who leave without being seen are not at increased risk of adverse events as they tend to be younger, less sick, and might seek care at another time; and patients in departments with higher annual rates of patient leaving without being seen are at greater risk of adverse events as these rates might reflect departmental inefficiency and dysfunction.

\section{METHODS}

\section{Setting and participants}

We conducted a retrospective cohort study of all emergency department patients in fiscal years 2003-7 (April 2003-March 2008) in Ontario, Canada (population 12 million). We ended enrolment on 28 February 2008 so we could assess seven day outcomes to 7 March 2008 . Records on hospital admissions are found in the fiscal year of the date of discharge so we allowed a three week period to include all admissions that would have been initiated in the first week of March 2008. We excluded emergency departments with a mean annual patient volume below the 25 th centile $(<13324)$ as they tend to have relatively few problems with waiting times ${ }^{14}$ : in our data, the median length of stay was one hour and the rate of leaving without being seen was less than $1 \%$. We included patients whose emergency department visit resulted in being either "seen and discharged" or "left without being seen." Left without being seen includes all patients who left before or after triage by a nurse (which typically happens before registration) but before further assessment by a physician; it excludes those who left after seeing a physician either without treatment or against medical advice. We included multiple visits by the same patient; when a patient had two visits within 30 days, however, we selected the earlier visit as these were potentially related.

\section{Study exposures}

The exposures of interest were mean emergency department level length of stay by shift, emergency department level annual rate of patients leaving without being seen, and patient level rate of leaving without being seen. The annual rate of patients who leave without being seen is a currently recommended national performance measure. ${ }^{11}$ How long patients spend in the emergency department is a good proxy for departmental crowding. ${ }^{1516}$ It has face validity given that it is an important determinant of patients' satisfaction, ${ }^{17}$ is patient focused, is readily calculated and understood, and is considered the "criterion standard" for the measurement of throughput and crowding. ${ }^{16}$ Length of stay was calculated separately for each emergency department and shift on each day as the mean length of stay from time of triage or registration, whichever came first, to final emergency department disposition (discharge or admission to an inpatient ward) of all patients who did not leave without being seen who presented at that department during that shift with a similar severity of illness based on triage score, including those admitted and waiting for beds in the hospital. Triage is done by nursing staff as soon as the patient arrives at the department to determine priority and degree of urgency. All Ontario emergency departments use a common, validated five level triage tool, the Canadian triage and acuity scale, with common training programmes for triage nurses. ${ }^{18}$ Triage scores were grouped as high acuity (1-3: resuscitation, emergent, and urgent) and low acuity (4-5: less urgent and nonurgent). If no similarly triaged patients presented during the shift, length of stay was calculated based on patients of the same category grouping (1-3 or 4-5) in the same department shift during the previous week (68 $899(0.47 \%)$ patients) or, in a few cases, in the same category grouping during the previous week $946(0.01 \%)$ patients). Triage data were unavailable for a small proportion of patients $(5030,0.82 \%)$ who left without being seen (these patients leave the department before even being triaged). We excluded them from the main analyses. We chose mean length of stay over median as median length of stay tends to be less subject to variability, which we believe might be important in affecting shift level variations in processes of care. The mean is also a better measure to identify shifts with extremes in length of stay, such as when numerous admitted patients are kept, or boarded, in the emergency department, a factor known to be associated with crowding..$^{19}$ Our measure of length of stay did not represent the length of stay for the individual patient but reflected the average waiting time for similar patients presenting during the same shift in which the patient presented.

\section{Main outcome measures}

We linked records of visits to emergency departments with outpatient and subsequent emergency department visits, hospital admissions, and death in the seven days after the index visit. We defined adverse events as death or a hospital admission within seven days after leaving the emergency department. This time frame was chosen to ensure that subsequent events were related to the index visit; others have observed that more than $80 \%$ of adverse events after discharge from an emergency department occur within the first 10 days. ${ }^{20}$

\section{Data sources}

Patients' records were linked with unique anonymised encrypted identifiers across multiple population based health administrative databases in Ontario containing information on all publicly insured, medically necessary hospital and physician services. Emergency department visits were identified through the national ambulatory care reporting system (NACRS); admission to hospital through the discharge abstract database; outpatient physician billings from the Ontario 
Table 1/Characteristics of patients who visited Ontario emergency departments according to whether patients left without being seen or were seen and discharged, 1 April 2003 to 28 February 2008. Figures are numbers (percentages)

Left without being seen $(n=617011)$

Seen and discharged $(n=13934$ 542)

Age group (years):

\begin{tabular}{|c|c|c|}
\hline$<1$ & $16269(2.6)$ & $331629(2.4)$ \\
\hline $1-9$ & 90339 (14.6) & 1807289 (13.0) \\
\hline $10-17$ & $59961(9.7)$ & $1427426(10.2)$ \\
\hline $18-39$ & $247705(40.2)$ & $4329599(31.1)$ \\
\hline $40-64$ & $156179(25.3)$ & $3981281(28.6)$ \\
\hline $65-75$ & $26210(4.3)$ & $1017965(7.3)$ \\
\hline$>75$ & 20348 (3.3) & $1039353(7.5)$ \\
\hline \multicolumn{3}{|l|}{ Sex: } \\
\hline Female & $317090(51.4)$ & $7122253(51.1)$ \\
\hline Male & $299921(48.6)$ & $6812289(48.9)$ \\
\hline \multicolumn{3}{|l|}{ Fifth of neighbourhood income: } \\
\hline 1 (lowest) & $158955(25.8)$ & $3205169(23.0)$ \\
\hline 2 & $131093(21.3)$ & $2884051(20.7)$ \\
\hline 3 & $118451(19.2)$ & $2738726(19.7)$ \\
\hline 4 & $109985(17.8)$ & $2671352(19.2)$ \\
\hline 5 (highest) & $95745(15.5)$ & $2376139(17.1)$ \\
\hline Missing & $2782(0.45)$ & $59105(0.42)$ \\
\hline \multicolumn{3}{|l|}{ Community type: } \\
\hline Urban & $485956(78.8)$ & $9839303(70.6)$ \\
\hline Semi-rural & $116145(18.8)$ & $3470646(24.9)$ \\
\hline Rural & $7502(1.2)$ & $381554(2.7)$ \\
\hline Missing & $7408(1.2)$ & $243039(1.7)$ \\
\hline \multicolumn{3}{|l|}{ CTAS category (score): } \\
\hline Resuscitation (1) & $70(0.01)$ & $16785(0.12)$ \\
\hline Emergent (2) & $23115(3.8)$ & $1283603(9.2)$ \\
\hline Urgent (3) & $238739(38.7)$ & $5343306(38.4)$ \\
\hline Less urgent (4) & $287858(46.7)$ & $6133737(44.0)$ \\
\hline Non-urgent (5) & $62199(10.1)$ & $1157111(8.3)$ \\
\hline Missing & $5030(0.82)$ & $0(0.0)$ \\
\hline \multicolumn{3}{|l|}{ Chief complaint at index visit: } \\
\hline Injury/trauma/musculoskeletal problems & $179325(29.1)$ & $5151619(37.0)$ \\
\hline Mental health & $16796(2.7)$ & $220520(1.6)$ \\
\hline Gastrointestinal & $85642(13.9)$ & $1452288(10.4)$ \\
\hline Chest pain & $27387(4.4)$ & $758331(5.4)$ \\
\hline Shortness of breath/asthma & $23582(3.8)$ & $475221(3.4)$ \\
\hline Upper respiratory tract infection/otitis media & $57066(9.3)$ & $1343556(9.6)$ \\
\hline Fever & $27825(4.5)$ & $461119(3.3)$ \\
\hline Headache/neurological & $44787(7.3)$ & $885617(6.4)$ \\
\hline Skin & $28561(4.6)$ & $663645(4.8)$ \\
\hline Genitourinary/obstetric & $21087(3.4)$ & $588539(4.2)$ \\
\hline Administrative issue & $37306(6.1)$ & $486972(3.5)$ \\
\hline General signs and symptoms & $30702(5.0)$ & $532381(3.8)$ \\
\hline Other & $36945(6.0)$ & $914734(6.6)$ \\
\hline \multicolumn{3}{|c|}{ No of visits to emergency department in previous year: } \\
\hline 0 & $347521(56.3)$ & $7864209(56.4)$ \\
\hline 1 & $132775(21.5)$ & 3009193 (21.6) \\
\hline 2 & 60064 (9.7) & $1367211(9.8)$ \\
\hline$\geq 3$ & $76651(12.4)$ & 1693929 (12.2) \\
\hline
\end{tabular}

CTAS=Canadian triage and acuity scale. health insurance plan (OHIP); and deaths from a population based registry of all legal residents in Ontario. Neighbourhood income and community type were derived from Statistics Canada 2001 census estimates.

\section{Statistical analyses}

We used conditional logistic regression models, conditioning on emergency department as fixed effects, to analyse the effects of leaving without being seen at the patient level and length of stay by shift on adverse events. This allowed us to assess the effects of longer versus shorter waiting times within the same department, controlling for other factors. ${ }^{21}$ As these models cannot include factors at the level of the emergency department, we used unconditional logistic regression to test the association between annual rates of leaving without being seen, measured at the department level, and adverse events in patients; in these latter models, we used generalised estimating equation models to account for clustering of patients' outcomes within emergency departments ${ }^{22}$ and controlled for other department characteristics (teaching status and annual patient volume). All models included length of stay and used the patient as the unit of analysis. This allowed us to control for individual risk factors and permitted inferences to outcomes for individual patients, even for exposures measured at the level of the emergency department. Models controlled for patients' age group (0-1, 1-9, 10-17, 18-39, 40-65, 66$75,>75$ years), sex, calendar month, weekend/holiday versus weekday, time of day (daytime (0801-1600), evening (1601-2400), night (2401-0800)), income fifth of the patients' neighbourhood (including missing) and community type (rural, urban, missing), number of visits to an emergency department in the past year, and main complaint. The department level model for patients who left without being seen also controlled for mean time to physician for similarly triaged patient who did not leave without being seen during the same shift, measured as time from triage to being seen by a physician; we did not include this in the model testing leaving without being seen at the patient level as it is in the causal pathway of patients leaving without being seen. All analyses were stratified by Canadian triage and acuity scale category (1-3 (high acuity) and 4-5 (low acuity)). Statistical tests were computed at the $\mathrm{P}<0.05$ and were two sided. We did not include the patient's own length of stay as a separate variable in the models as we were investigating shift characteristics; however, it was included in the shift level calculation of length of stay in the emergency department. Missing data were handled as follows: missing fifth of neighbourhood income relates to the instability of the population in any given dissemination area (for instance, areas with high numbers of university students) and as such this category was kept separate and those patients were included. Similarly, missing data on community type reflect recent changes in neighbourhood boundaries that have not yet been mapped adequately by Statistics Canada and these patients were also analysed as a separate category. Missing 
Table 2 |Characteristics of shifts and emergency departments among patients who left without being seen or were seen and discharged, 1 April 2003 to 28 February 2008. Figures are numbers (percentages)

Left without being seen Seen and discharged

$(n=617011)$

$(n=13934542)$

Mean length of stay (hours) in emergency department in patients who did not leave without being seen*:

\begin{tabular}{lcc}
\hline$\ll 1$ & $5232(0.85)$ & $715656(5.1)$ \\
\hline $1-<2$ & $51114(8.3)$ & $2914393(20.9)$ \\
\hline $2-<3$ & $109743(17.8)$ & $2986808(21.4)$ \\
\hline $3-<4$ & $120522(19.5)$ & $2525333(18.1)$ \\
\hline $4-<5$ & $112082(18.2)$ & $2012096(14.4)$ \\
\hline $5-<6$ & $92610(15.0)$ & $1360140(9.8)$ \\
\hline$\geq 6$ & $125708(20.4)$ & $1420099(10.2)$ \\
\hline Missing & $0(0.0)$ & $17(0.0)$ \\
\hline
\end{tabular}

Mean time to assessment (hours) by doctor in patients who did not leave without being seen*:

\begin{tabular}{|c|c|c|}
\hline$<1$ & $62360(10.1)$ & $4768258(34.2)$ \\
\hline $1-<2$ & $217294(35.2)$ & $5920050(42.5)$ \\
\hline $2-\ll 3$ & $198964(32.3)$ & $2340187(16.8)$ \\
\hline $3-<4$ & 91867 (14.9) & $637090(4.6)$ \\
\hline$\geq 4$ & $44593(7.2)$ & $188783(1.4)$ \\
\hline Missing & $1933(0.31)$ & $80174(0.58)$ \\
\hline \multicolumn{3}{|l|}{ Time of day (shift): } \\
\hline Day (8 am to $4 \mathrm{pm})$ & $222279(36.0)$ & $6353908(45.6)$ \\
\hline Evening ( $4 \mathrm{pm}$ to midnight) & $297894(48.3)$ & $5630132(40.4)$ \\
\hline Night (midnight to 8 am) & $96838(15.7)$ & $1950502(14.0)$ \\
\hline \multicolumn{3}{|l|}{ Day of week: } \\
\hline Weekday & $436357(70.7)$ & 9484359 (68.1) \\
\hline Weekend/holiday & $180654(29.3)$ & $4450183(31.9)$ \\
\hline \multicolumn{3}{|l|}{ Hospital type: } \\
\hline Small & $28675(4.7)$ & $1164261(8.4)$ \\
\hline Community & $483852(78.4)$ & $10645901(76.4)$ \\
\hline Teaching & $104484(16.9)$ & $2124380(15.3)$ \\
\hline \multicolumn{3}{|c|}{ Average annual departmental volume: } \\
\hline $13324-<26000$ & 73144 (11.9) & $2766442(19.9)$ \\
\hline $26000-<45000$ & $220148(35.9)$ & 4579504 (32.9) \\
\hline $45000-89792$ & $323719(52.5)$ & $6588596(47.3)$ \\
\hline
\end{tabular}

*Within same shift in same department.

mean time to physician was also retained as its own category. All analyses were performed with SAS for UNIX, version 9.2.1 (SAS Institute, Cary, NC). Conditional logistic models used the procedure PROC LOGISTIC with a STRATA statement and included fixed effects for each emergency department.

\section{RESULTS}

There were 21925275 visits during the study period, of which 1487094 were to emergency departments with low volume. Of the remaining 20438181 visits in 125 departments, 17771926 (87\%) patients left without being seen or were seen and discharged. We excluded 3220373 visits within 30 days of a previous visit, leaving 14551553 visits, of which 617011 were in patients who left without being seen and 13934542 were in patients who were seen and discharged.

The median (interquartile range) annual rate of those who left without being seen was 3.6\% (2.5-4.8\%). These patients were younger, had slightly lower acuity triage scores (table 1), and attended more often during the evening shift and during shifts when mean length of stay and time to see a physician were longer (table 2). They were more likely to live in urban areas (table 1) and attend higher volume departments (table 2).

Absolute rates of death and admission were much higher in high than in low acuity patients, and were lower in those who left without being seen than in those who were seen and discharged (table 3). Rates of subsequent visits to emergency departments and physician offices or outpatient clinics were higher in patients who left without being seen. In all patients rates of death and admission to hospital increased with increasing length of stay in an emergency department (table 4).

In multivariable analyses, the risk of death increased incrementally with each additional hour of mean waiting time per shift: the adjusted odds ratios (95\% confidence interval) for death and admission were 1.79 (1.24 to 2.59 ) and 1.95 (1.79 to 2.13 ), respectively, among high acuity patients (fig 1), and 1.71 (1.25 to 2.35 ) and 1.66 (1.56 to 1.76 ), respectively, among low acuity patients (fig 2) for mean length of stay of $\geq 6$ hours compared with $<1$ hour.

The adjusted risk of death was significantly lower for patients who left without being seen compared with those who were seen and discharged. The adjusted rate of hospital admission for patients who left without being seen was lower for low acuity patients (fig 2 ) but similar to those who were seen and discharged for high acuity patients (fig 1). The annual rate of leaving without being seen at the emergency department level was not associated with a clinically important risk of either death or admission (adjusted odds ratios 1.00 (0.97 to 1.02 ) for death and 0.98 (0.97 to 0.99$)$ for admission in high acuity patients; 1.03 (0.99 to 1.06 ) for death and 0.99 (0.98 to 1.00) for admission in low acuity patients).

Death was more likely in the three oldest age groups than in patients aged 18-39 for both high and low acuity patients. Admissions were higher in the three oldest age groups and in those aged under 1 year compared with those aged 18-39. Full model results can be found in the appendix on bmj.com.

\section{DISCUSSION}

Patients presenting to emergency departments during shifts with long mean waiting times might be at increased risk of death and admission in the subsequent seven days, regardless of acuity on presentation. As mean length of stay increases, so does the risk of subsequent adverse events. Although the absolute risk of death and admission for low acuity patients is smaller, the relative impact of increased length of stay seems to be slightly higher.

These results come from a large population based study that explored the safety implications of average waiting times in emergency departments among patients who go home after their visit. Such patients represented about $90 \%$ of the total in our study and comprise the majority in all emergency department systems in developed countries. We were able to 
Table 3| Outcomes among emergency department patients who were seen and discharged or left without being seen according to acuity level (Canadian triage and acuity scale) at presentation 1 April 2003 to 28 February 2008. Figures are numbers (percentages)

\begin{tabular}{|c|c|c|c|c|c|c|}
\hline \multirow{2}{*}{$\begin{array}{l}\text { Outcomes within seven } \\
\text { days after index visit to } \\
\text { emergency department }\end{array}$} & \multicolumn{2}{|c|}{ High acuity (score 1-3) } & \multicolumn{2}{|c|}{ Low acuity (score 4-5) } & \multicolumn{2}{|c|}{ All patients* } \\
\hline & $\begin{array}{l}\text { Left without being } \\
\text { seen }(n=261924)\end{array}$ & $\begin{array}{l}\text { Seen and discharged } \\
\quad(n=6643694)\end{array}$ & $\begin{array}{l}\text { Left without being } \\
\text { seen }(n=350057)\end{array}$ & $\begin{array}{l}\text { Seen and discharged } \\
\quad(n=7290848)\end{array}$ & $\begin{array}{l}\text { Left without being } \\
\text { seen }(n=617011)\end{array}$ & $\begin{array}{l}\text { Seen and discharged } \\
\quad(n=13934542)\end{array}$ \\
\hline Death & $123(0.047)$ & $8502(0.13)$ & $56(0.016)$ & $1998(0.027)$ & $181(0.029)$ & $10500(0.075)$ \\
\hline Admission to hospital & $6784(2.6)$ & 190749 (2.9) & $2782(0.80)$ & $61157(0.84)$ & $9651(1.6)$ & 251906 (1.8) \\
\hline Return visit & 36107 (13.8) & $640521(9.6)$ & $50792(14.5)$ & $493472(6.8)$ & $87943(14.3)$ & $1133993(8.1)$ \\
\hline Return higher acuity $†$ visit & $4138(1.6)$ & $62746(0.94)$ & $18104(5.2)$ & 190059 (2.6) & $22242(3.6)$ & $252805(1.8)$ \\
\hline Any outpatient clinic visit & $102347(39.1)$ & $2407869(36.2)$ & $108972(31.1)$ & $1766947(24.2)$ & $212745(34.5)$ & $4174816(30.0)$ \\
\hline
\end{tabular}

*Does not equal sum of high and low acuity patients because of missing data in level of acuity.

†Decrease in Canadian triage and acuity scale of 1 point or more compared with index emergency department visit.

show an increase in the risk of both mortality and admission to hospital associated with presenting during shifts with long mean lengths of stay. In contrast, patients who left without being seen were not at increased risk of adverse events compared with those who were seen and discharged, nor were patients who attend departments with high rates of leaving without being seen. Patients who left without being seen had slightly higher rates of follow-up with primary care physicians and return visits to the emergency department than those who were seen and discharged, which could have helped to mitigate adverse events. Some could have problems that resolve while they are waiting to be seen.

\section{Comparison with other studies}

Patients who leave without being seen have long been considered at risk of adverse events, ${ }^{23-25}$ but previous studies were small, single centre, or did not examine mortality. ${ }^{23-25}$ The absence of increased risk should be reassuring to emergency department clinicians and administrators, who have struggled with the uncertainty surrounding outcomes in such patients. Our results suggest that individual patients who leave

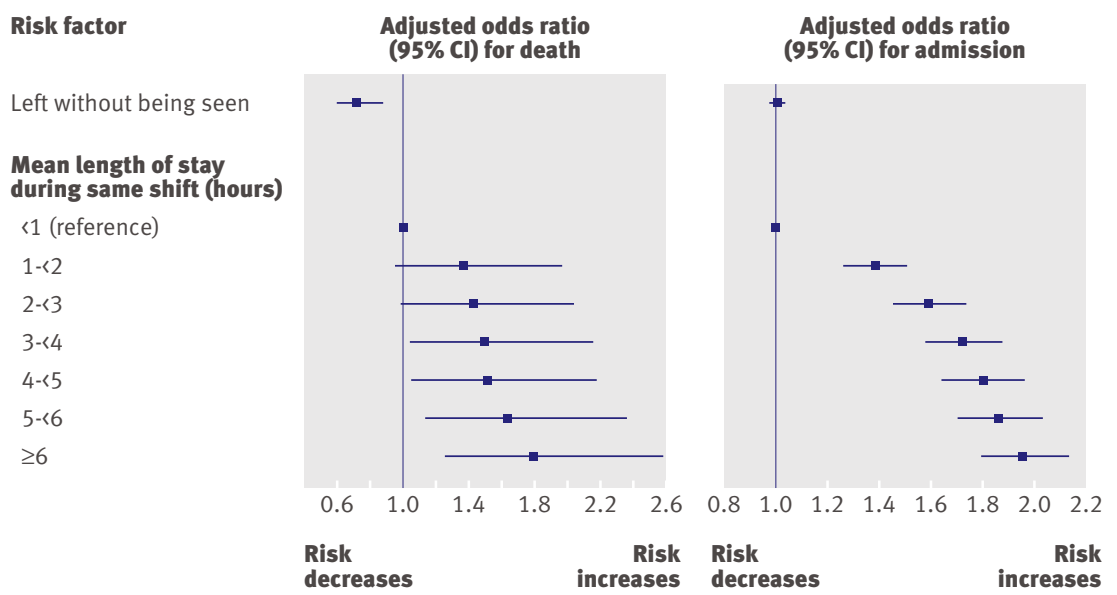

Fig 1 Adjusted odds ratios (95\% confidence intervals) for death and admission to hospital within seven days of emergency department visit among all non-admitted (seen and discharged and left without being seen) high acuity patients (Canadian triage and acuity scale levels 1 to 3). Odds ratios adjusted for triage level, age group, sex, calendar month, income fifth, urban/rural community, No of visits to emergency department in previous year, chief complaint, time/day of shift without being seen are not at increased risk of adverse events, and that the emergency department level rate of leaving without being seen, taken alone, cannot be seen as a measure of patient safety.

On the other hand, our results suggest that presenting during shifts with a long mean length of stay could have serious safety implications for patients. We tested for associations between mean length of stay and adverse events both between and within institutions. Our findings build on previous research that has been largely restricted to patients admitted from the emergency department or with high acuity illnesses in tertiary care hospitals. An Australian study showed a 35\% increase in 10 day mortality associated with higher emergency department occupancy among all emergency department patients in a single teaching hospital but only identified deaths occurring in the same hospital. ${ }^{26}$ Another study showed that mortality at two, seven, and 30 days was related to both emergency department and hospital crowding, but examined only patients admitted from the emergency department in three tertiary care hospitals. ${ }^{27}$ Studies of patients with acute myocardial infarction ${ }^{9}$ and pneumonia ${ }^{10}$ have found lower rates of evidence based time sensitive treatments associated with waiting times and crowding in emergency departments. These studies point to potential mechanisms of harm in specific high acuity illnesses, while other studies have shown delays in more general processes of care associated with emergency department crowding such as time to interpretation of diagnostic imaging. ${ }^{28}$

It is unlikely that delays in treatment alone account for adverse outcomes in the patients we studied, all of whom left the emergency department, most after being seen and discharged, implying that the intended care was completed. It is possible, however, that during shifts with long mean waiting times important processes of care are impeded and decision making altered. ${ }^{1223}$ Examples might include reluctance to order time consuming tests or consultations and shortened observation periods (both of which could increase missed diagnoses), incomplete treatment, or inadequate planning and communication of care after discharge. The increased risk of adverse events for low acuity patients suggests that processes might be more likely to break down if patients are thought to be low 


Risk factor
Left without being seen
Mean length of stay
during same shift (hours)
$<1$ (reference)
$1-<2$
$2-\ll 3$
$3-<4$
$4-<5$
$5-<6$
$\geq 6$

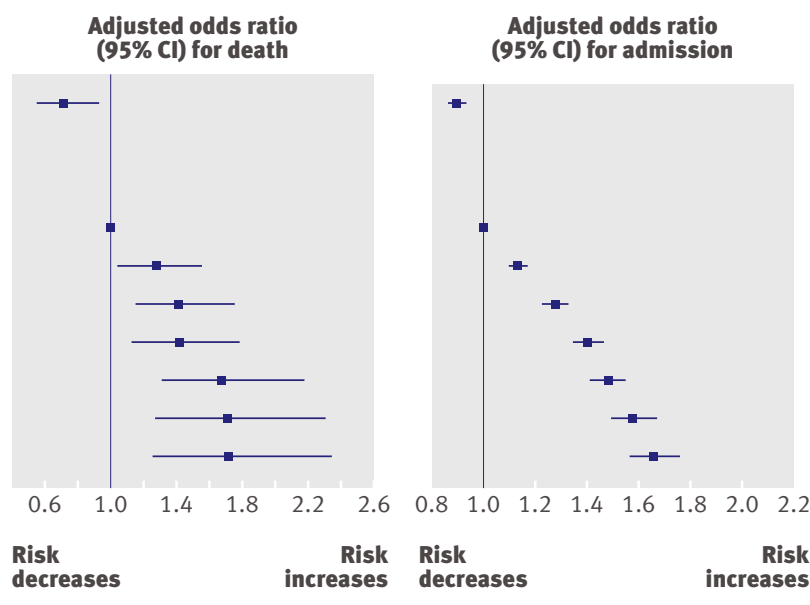

Fig 2 | Adjusted odds ratios (95\% confidence intervals) for death and admission to hospital within seven days of emergency department visit among all non-admitted (seen and discharged and left without being seen) low acuity patients (Canadian triage and acuity scale levels 4 to 5). Odds ratios adjusted for triage level, age group, sex, calendar month, income fifth, urban/rural community, No of visits to emergency department in previous year, chief complaint, time/day of shift this goal. Policies to reduce length of stay are aligned with the Institute of Medicine's patient safety initiative ${ }^{4}$ and could decrease important adverse events. The increasing risk to patients associated with increasing length of stay in an emergency department suggests that any reductions, regardless of magnitude, could benefit a wide array of patients. In our analysis, reducing mean length of stay by an average of one hour could have potentially decreased the number of deaths in our study in higher acuity patients $(8625)$ by 558 $(6.5 \%)$ and in lower acuity patients (2054) by 261 $(12.7 \%)$. Further research needs to evaluate whether these benefits are in fact seen when waiting times are decreased.

\section{Strengths and limitations of study}

Strengths of our study include the large number of patients, inclusion of community and tertiary hospitals, linkage across healthcare sectors, and no loss to follow-up. Administrative health data are subject to some inaccuracies, but we limited our analyses to highly reliable data elements. ${ }^{33}$ Our findings might not be generalisable to other healthcare systems. The findings around patients who leave without being seen might not be the same in systems such as in the United States without universal access to other healthcare services, although we found that patients who left without being seen were only slightly more likely than those who were seen and discharged to seek office based outpatient care after an emergency department visit, suggesting that access to primary care for patients who leave without being seen in Ontario was not a major factor in limiting adverse events. A lower admission threshold in other systems might mean fewer high risk patients being discharged home and thus avoiding need for subsequent admission, although our results indicate the risk associated with long waiting times extends to low acuity patients as well, nearly all of whom are discharged home in all systems. Ontario's rate of use of emergency departments is comparable with that in other jurisdictions, ${ }^{11}$ suggesting case mix is similar, although some other countries have reported higher rates of patients who leave without being seen. ${ }^{34}$ The organisation of care within emergency departments in Canada is similar to that in most developed countries, and crowding has become a common

Table 4|Outcomes among emergency department patients who were seen and discharged or left without being seen according to mean length of stay of similar patients in emergency department on same shift, 1 April 2003 to 28 February 2008

\begin{tabular}{|c|c|c|c|c|c|c|}
\hline \multirow[b]{2}{*}{ Mean length of stay (hours) } & \multicolumn{3}{|c|}{ High acuity (Canadian triage and acuity scale $1-3$ ) } & \multicolumn{3}{|c|}{ Low acuity (Canadian triage and acuity scale 4-5) } \\
\hline & No & Died (\%) & Admitted (\%) & No & Died (\%) & Admitted (\%) \\
\hline$<1$ & 34087 & 0.094 & 2.23 & 685544 & 0.020 & 0.67 \\
\hline $1-<2$ & 330507 & 0.120 & 2.82 & 2636122 & 0.023 & 0.74 \\
\hline $2-\ll 3$ & 888838 & 0.110 & 2.78 & 2203178 & 0.026 & 0.83 \\
\hline $3-<4$ & 1456504 & 0.112 & 2.76 & 1190722 & 0.029 & 0.95 \\
\hline $4-<5$ & 1593044 & 0.119 & 2.83 & 529281 & 0.039 & 1.06 \\
\hline $5-46$ & 1238144 & 0.132 & 2.90 & 214925 & 0.043 & 1.18 \\
\hline$\geq 6$ & 1364478 & 0.151 & 3.04 & 181132 & 0.045 & 1.24 \\
\hline
\end{tabular}




\section{WHAT IS KNOWN ON THIS TOPIC}

Long waiting times in the emergency department are a widespread problem in developed countries and known to be associated with delays in care and poor outcomes for sick patients who require time sensitive care or admission, or both

Most patients go home from the emergency department, usually after medical assessment, though up to $10 \%$ might leave without being seen by a physician

\section{WHAT THIS STUDY ADDS}

For every extra hour of mean length of stay in an emergency department, in similar patients at the time of presentation, there is an association with increased seven day mortality and admission to hospital in those who are discharged home or leave without being seen

Patients who leave without being seen do not have a higher adjusted risk of adverse outcomes compared with patients who are seen and discharged

Waiting times have important implications for patients who attend emergency departments, most of whom go home after their visit

problem in many systems. Finally, as an observational study it is possible the association between waiting times and adverse events might have been subject to confounding. Although we have shown that the association exists within as well as between emergency departments, there could be local factors at play during shifts with long waiting times, such as differences in staffing, that might be unmeasured drivers of adverse events.

\section{Conclusions and policy implications}

Waiting times in emergency departments, as indicated by prolonged length of stay, are associated with important safety outcomes for patients, even for those who are well enough to leave the emergency departmentthe vast majority of patients. Contrary to popular belief, leaving without being seen was not associated with a higher risk of adverse events in the short term. Reducing adverse events attributable to long waiting times among patients who go home is probably best achieved by reducing the overall length of stay in emergency departments for all patients, rather than targeting patients who leave without being seen for review or follow-up. Further research on patient safety should evaluate whether quality improvement and performance measurement initiatives prioritising waiting times in emergency departments result in reductions of adverse events.

We thank Kinwah Fung for help with the data analysis and Joel Ray for help in preparing the manuscript.

Contributors: AG was responsible for conception, design, analysis,

funding, interpretation, drafting of article, and critical revision. MS and TS were involved in design, analysis, interpretation, and critical revision. MV was responsible for design, analysis, interpretation, drafting of article, and critical revision. AG is guarantor. All authors had full access to the data and take responsibility for its integrity and the accuracy of the analysis.

Funding: This study was supported by a grant from the Ontario Ministry of Health and Long Term Care and by the Institute for Clinical Evaluative Sciences, which is funded by an annual grant from the Ministry of Health and Long Term Care. The funding source had no involvement in the study design, analysis, interpretation or decision to submit this work.

Competing interests: All authors have completed the Unified Competing Interest form at www.icmje.org/coi_disclosure.pdf (available on request from the corresponding author) and declare: AG received financial support from the Ontario Ministry of Health and Long Term Care for the submitted work and she and MS receive salary support from the Canadian Institute for Health Research; the authors have no financial relationships with any organizations that might have an interest in the submitted work in the previous 3 years; no other relationships or activities that could appear to have influenced the submitted work. Ethical approval: This study was approved by the research ethics board of Sunnybrook Health Sciences Centre.

Data sharing: The technical appendix, dataset creation plan/protocol and statistical code are available from the corresponding author at astrid.guttmann@ices.on.ca.

1 Bond K, Ospina MB, Blitz S, Afilalo M, Campbell S, Bullard M, et al. Frequency, determinants and impact of overcrowding in emergency departments in Canada: a national survey. Healthc $Q$ 2007; 10:32-40

2 Fatovich DM, Nagree Y, Sprivulis P. Access block causes emergency department overcrowding and ambulance diversion in Perth, Western Australia. Emerg Med / 2005;22:351-4.

3 Locker T, Mason S, Wardrope J, Walters S. Targets and moving goal posts: changes in waiting times in a UK emergency department. Emerg Med J 2005;22:710-4.

4 Institute of Medicine of the National Academies, Committee on the Future of Emergency Care in the United States Health System. Hospital-based emergency care: at the breaking point. National Academies Press, 2006.

5 Alberti G. Transforming emergency care in England. Department of Health, 2004.

6 Ontario Ministry of Health and Long Term Care. McGuinty government launches public reporting of time spent in the ER. 19 February 2009. www.health.gov.on.ca/english/media/news_releases/archives/ nr_09/feb/nr_20090219.html.

7 Peatling S. Rudd promises to halve waiting times. Sudbury Morning Herald 11 April 2010.

8 Topping A, Campbell D. Waiting targets for accident and emergency to be scrapped. Guardian 10 June 2010.

9 Diercks DB, Roe MT, Chen AY, Peacock WF, Kirk JD, Pollack CV Jr, et al. Prolonged emergency department stays of non-ST-segmentelevation myocardial infarction patients are associated with worse adherence to the American College of Cardiology/American Heart Association guidelines for management and increased adverse events. Ann Emerg Med 2007;50:489-96.

10 Pines JM, Localio AR, Hollander JE, Baxt WG, Lee H, Phillips C, et al. The impact of emergency department crowding measures on time to antibiotics for patients with community-acquired pneumonia. Ann Emerg Med 2007; 50:510-6.

11 Pitts SR, Niska RW, Xu J, Burt CW. National Hospital Ambulatory Medical Care Survey: 2006 emergency department summary. National Health Statistics Reports, No 7. US National Center for Health Statistics, 2008.

12 Mohsin M, Forero R, leraci S, Bauman AE, Young L, Santiano N. A population follow-up study of patients who left an emergency department without being seen by a medical officer. Emerg MedJ 2007;24:175-9.

13 National Quality Forum. NQF endorses measures to address care coordination and efficiency in hospital emergency departments. 29 October 2008. http://urgentmatters.org/media/file/NQF\%20Press $\% 20$ Release.pdf.

14 Bell R, Willett J, Oliver J, an Expert Working Group Reporting to the Physician Hospital Care Committee. Improving access to emergency care: addressing system issues. Ontario Hospital Association, the Ontario Medical Association and the Ontario Ministry of Health and Long Term Care, 2006.

15 McCarthy ML, Zeger SL, Ding R, Levin SR, Desmond JS, Lee J, et al. Crowding delays treatment and lengthens emergency department length of stay, even among high-acuity patients. Ann Emerg Med 2009;54:492-503.

16 Asplin BR. Measuring crowding: time for a paradigm shift. Acad Emerg Med 2006;13:459-61.

17 Bernstein SL, Aronsky D, Duseja R, Epstein S, Handel D, Hwang U, et al. The effect of emergency department crowding on clinically oriented outcomes. Acad Emerg Med 2009;16:1-10.

18 Murray MJ, Bullard M, Grafstein E. Revisions to the Canadian Emergency Department Triage and Acuity Scale Implementation Guidelines. Can J Emerg Med 2004;6:421-7.

19 Schull MJ, Lazier K, Vermeulen M, Mawhinney S, Morrison LJ. Emergency department contributors to ambulance diversion: a quantitative analysis. Ann Emerg Med 2003;41:467-76.

20 Forster AJ, Rose NG, van Walraven C, Stiell I. Adverse events following an emergency department visit. Qual Saf Health Care 2007;16:17-22.

21 Berlin JA, Kimmel SE, Ten Have TR, Sammel MD. An empirical comparison of several clustered data approaches under 
confounding due to cluster effects in the analysis of complications of coronary angioplasty. Biometrics 1999;55:470-6.

22 Zeger SL, Liang KY. Longitudinal data analysis for discrete and continuous outcomes. Biometrics 1986;42:121-30.

23 Baker DW, Stevens CD, Brook RH. Patients who leave a public hospital emergency department without being seen by a physician. Causes and consequences. JAMA 1991;266:1085-90.

24 Bindman AB, Grumbach K, Keane D, Rauch L, Luce JM. Consequences of queuing for care at a public hospital emergency department. JAMA 1991;266:1091-6.

25 Browne GJ, McCaskill ME, Giles H, Lam LT, Fasher BJ, Exley B. Paediatric walk-out patients: characteristics and outcomes. J Paediatr Child Health 2001;37:235-9.

26 Richardson DB. Increase in patient mortality at 10 days associated with emergency department overcrowding. Med J Aust 2006;184:213-6.

27 Sprivulis PC, Da Silva JA, Jacobs IG, Frazer AR, Jelinek GA. The association between hospital overcrowding and mortality among patients admitted via Western Australian emergency departments. Med J Aust 2006;184:208-12.

28 Mills AM, Baumann BM, Chen EH, Zhang KY, Glaspey LJ, Hollander JE, et al. The impact of crowding on time until abdominal CT interpretation in emergency department patients with acute abdominal pain. Postgrad Med 2010;122:75-81.
29 Atzema CL, Austin PC, Tu JV, Schull MJ. Emergency department triage of acute myocardial infarction patients and the effect on outcomes. Ann Emerg Med 2009;53:736-45.

30 Moskop JC, Sklar DP, Geiderman JM, Schears RM, Bookman KJ. Emergency department crowding, part 2-barriers to reform and strategies to overcome them. Ann Emerg Med 2009;53:612-7.

31 Holroyd BR, Bullard M), Latoszek K, Gordon D, Allen S, Tam S, et al. Impact of a triage liaison physician on emergency department overcrowding and throughput: a randomized controlled trial. Acad Emerg Med 2007;14:702-8.

32 Bond K, Ospina MB, Blitz S, Friesen C, Yoon P, Curry G, et al. Interventions to reduce overcrowding in emergency departments. Technology report No 67.4. Canadian Agency for Drugs and Technologies in Health, 2006.

33 Canadian Institute for Health Information. $\mathrm{ClHI}$ data quality study of emergency department visits for 2004-2005: volume II of IV—main study findings. ClHI, 2008.

34 Australian Government Department of Health and Ageing. The state of our public hospitals, June 2008 report. Part 4: our emergency departments. Australian Government Department of Health and Ageing, 2008.

Accepted: 28 March 2011 